EGU21-9974, updated on 04 Mar 2021

https://doi.org/10.5194/egusphere-egu21-9974

EGU General Assembly 2021

(c) Author(s) 2021. This work is distributed under

the Creative Commons Attribution 4.0 License.

\title{
Spectral X-ray computed micro tomography: a tool for 3-dimensional chemical imaging
}

\author{
Jonathan Sittner ${ }^{1,2}$, Margarita Merkulova ${ }^{2}$, Jose Ricardo da Assuncao Godinho ${ }^{1}$, Axel Renno ${ }^{1}$, \\ Veerle Cnudde ${ }^{2,3}$, Marijn Boone ${ }^{4}$, Thomas De Schryver ${ }^{4}$, Denis Van Loo ${ }^{4}$, Antti Roine ${ }^{5}$, Jussi Liipo ${ }^{5}$, \\ Bradley Martin Guy ${ }^{6}$, and Stijn Dewaele ${ }^{2}$ \\ ${ }^{1}$ Helmholtz-Zentrum Dresden-Rossendorf, Analytics, Freiberg, Germany \\ ${ }^{2}$ PProGRess-UGCT, Geology Department, Ghent University, Ghent, Belgium \\ ${ }^{3}$ Department of Earth Sciences, Utrecht University, Utrecht, The Netherlands \\ ${ }^{4}$ TESCAN XRE, Ghent, Belgium \\ ${ }^{5}$ Metso-Outotec, Espoo, Finland \\ ${ }^{6}$ University of Johannesburg, Johannesburg, Republic of South Africa
}

Image-based analytical tools in geosciences are indispensable for the characterization of minerals, but most of them are limited to the surface of a polished plane in a sample and lack 3D information. X-ray micro computed tomography (micro CT) provides the missing 3D information of the microstructures inside samples. However, a major drawback of micro CT in the characterization of minerals is the lack of chemical information that makes mineral classification challenging.

Spectral X-ray micro computed tomography (Sp-CT) is a new and evolving tool in different applications such as medicine, security, material science, and geology. This non-destructive method uses a multi-pixel photon-counting detector (PCD) such as cadmium telluride (CdTe) in combination with a conventional CT scanner (TESCAN CoreTOM) to image a sample and detect its transmitted polychromatic X-ray spectrum. Based on the spectrum, elements in a sample can be identified by an increase in attenuation at specific K-edge energies. Therefore, chemically different particles can be distinguished inside a sample from a single CT scan. The method is able to distinguish elements with K-edges in the range from 25 to $160 \mathrm{keV}$, which applies to elements with Z > 48 (Sittner et al., 2020).

We present results from various sample materials. Different pure elements and element oxides were measured to compare the position of theoretical and measured K-edge energies. All measured K-edge energies are slightly above the theoretical value, but based on the results a correction algorithm could be developed. Furthermore, different monazite grains were investigated, which can be divided into two groups with respect to the content of different RE elements on the basis of the spectrum: La-Ce-rich and La-Ce-poor. In addition, samples from the Au-U Witwatersrand Supergroup demonstrate the potential applications of Sp-CT for geological samples. We measured different drill core samples from the Kalkoenkrans Reef at the Welkom 
Gold field. Sp-CT can distinguish gold, uraninite and galena grains based on their K-edge energies in the drill core without preparation.

Sittner, J., Godinho, J. R. A., Renno, A. D., Cnudde, V., Boone, M., De Schryver, T., Van Loo, D., Merkulova, M., Roine, A., \& Liipo, J. (2020). Spectral X-ray computed micro tomography: 3-dimensional chemical imaging. X-Ray Spectrometry, September, 1-14. 